\title{
Tangence
}

\section{Polar, scalaire et lecture}

\section{Bertrand Gervais}

Numéro 38, décembre 1992

Fiction policière et roman actuel

URI : https://id.erudit.org/iderudit/025742ar

DOI : https://doi.org/10.7202/025742ar

Aller au sommaire du numéro

Éditeur(s)

Tangence

ISSN

1189-4563 (imprimé)

1710-0305 (numérique)

Découvrir la revue

Citer cet article

Gervais, B. (1992). Polar, scalaire et lecture. Tangence, (38), 96-106. https://doi.org/10.7202/025742ar d'utilisation que vous pouvez consulter en ligne.

https://apropos.erudit.org/fr/usagers/politique-dutilisation/ 


\section{Polar, scalaire et lecture}

\section{Bertrand Gervais}

Dans la veine des moins bons romans policiers, je veux commencer par une confession. Je présente ici quelques-uns des résultats préliminaires d'une recherche collective sur l'acte de lecture $^{1}$. L'objectif de cette recherche était, à partir d'un corpus de romans policiers, de mettre à l'épreuve la notion de scalaire et de vérifier son impact sur l'acte de lecture. L'hypothèse développée était que des récits de complexités différentes prescrivent divers registres de lecture. Tous les textes ne se lisent pas de la même façon, tous ne requièrent pas les mêmes compétences de lecture. Certains récits, comme bien des romans populaires, ne mettent en jeu qu'un minimum de connaissances, étant fortement linéaires par exemple; d'autres, comme le nouveau roman ou les romans postmodernes, exigent des compétences spécifiques, avec leurs intrigues complexes, leur subversion des conventions narratives et littéraires, etc. Les lectures varient donc selon les exigences des récits lus: c'est l'hypothèse du scalaire ${ }^{2}$.

Pour tester cette hypothèse du scalaire, une série de récits aux exigences différentes a donc été constituée. Deux principes étaient

1 Cette recherche fait partie des travaux du GREL (Groupe de recherche en lecture) et les résultats ont été obtenus lors d'un séminaire de deuxième et troisième cycle, qui s'est tenu à l'UQAM en 1990-1991 et qui portait sur une sémiotique de la lecture. Un collectif, regroupant les contributions des principaux intervenants de ce séminaire, devrait paraître en 1992, dans la série de cahiers * Recherches et documents " du GREL.

2 Il ne s'agit pas par là de développer un système de gradation rigide, qui serait à toutes fins utiles inopérant, mais d'utiliser la notion de scalaire un peu à la manière de Claude Hagège, pour qui elle est avant tout - une échelle à transition souple entre les degrés n (L'bomme de paroles, Paris, Gallimard, Folio essais *, 1985, p. 183). Une seconde remarque s'impose. La perspective utilisée ne cherche pas à rendre compte de qualités intrinsèques du texte, elle ne cherche pas, par exemple, à retrouver les traces d'une littérarité, qui permettrait de séparer les textes et de leur imposer une valeur; au contraire, il s'agit de comparer des degrés de difficulté, d'identifier des problèmes de lecture, indépendamment de leur valeur. 
à la base de l'établissement de cette série: la verticalité (la série s'élabore à partir des discours les plus simples et les plus linéaires et se déploie vers les plus complexes) et la reconnaissance institutionnelle (les textes retenus sont ceux que l'institution, la critique littéraire, le monde de l'édition par ses collections et ses séries, ont classés comme littéraires, paralittéraires ou pour la jeunesse). Le roman policier a été choisi pour constituer la série à cause de son importance et de l'importance de sa production dans la littérature occidentale contemporaine. Comme en fait foi le thème de ce dossier, la forme policière n'est plus l'exclusivité d'un genre paralittéraire mais participe pleinement de la production actuelle.

Les textes étudiés ont été les suivants: Le roman d'Agatha d'Yves E. Arnau ${ }^{3}$; Le poison dans l'eau de Chrystine Brouillet ${ }^{4}$; le premier de la série des enquêtes de Maud Graham; Copies conformes de Monique LaRue 5 ; Douze suspects... pour un suicide de Pierre Saurel ${ }^{6}$; et, enfin, Meurtres à blanc de Yolande Villemaire 7 . Les uns appartiennent à la littérature populaire (Saurel), à la littérature pour la jeunesse (Arnau), à une collection de romans policiers un peu plus haut de gamme (Brouillet, dont le roman est paru dans la collection "Sueurs froides"), et les autres à la littérature proprement dite (LaRue, dont le roman a gagné le Grand Prix du livre de Montréal en 1990) et à une esthétique postmoderne (Villemaire). Tous ces polars cependant sont des textes québécois, qui font la preuve, à défaut d'une véritable tradition, de l'émergence d'une littérature policière nationale ${ }^{8}$.

3 Montréal, Éditions Pierre Tisseyre, série * Une aventure d'Edgar Allan, détective. (pour les jeunes de 10 à 12 ans), 1989, 137 p.

4 Éditions Denoël/Editions Lacombe, coll. . Sueurs froides *, 1987, 208 p.

5 Éditions Denoël/Éditions Lacombe, 1989, $190 \mathrm{p}$.

6 Montréal, Québec/Amérique, série - le Manchot *, 1983, 156 p.

7 Montréal, Le cadavre exquis, 1974,164 p. Le texte a été repris dans la collection : Typon.

$8 \mathrm{Ne}$ prendre que des romans québécois comme corpus était une façon, entre autres, de simplifier le problème des savoirs culturels, sociaux, symboliques, etc., qui ne manque pas de se poser dans toute problématisation de la lecture d'un texte. En puisant dans un répertoire québécois, on réduisait les difficultés liées à ces aspects de la lecture, considérant leur maîtrise simplement comme un acquis et ne cherchant pas, par conséquent, à développer cette dimension de l'acte. Cela n'aurait pas été aussi simple avec, par exemple, le roman noir américain des Dashiell Hammett, Raymond Chandler ou Ross Macdonald, qui se passe sur la côte ouest, ou encore le roman policier français de Léo Malet, qui joue à fond la carte parisienne, ou de René Belletto, basé à Lyon. 
98

Les différences entre ces polars ont été examinées de bien des façons: au niveau de leur mise en intrigue, des problèmes de cohérence et de synthèse, des personnages et de leur représentation, des interactions, de la spatialité et de la référence, des niveaux de lisibilité, des dialogues et du contrôle de l'information, de l'implicite, des incipits, des jeux de langage et encore de l'intertextualité, sur laquelle je m'arrêterai plus précisément ici. Je me contenterai de rendre compte d'une intertextualité ou interdiscursivité de surface, dite encore explicite. Le présupposé de mon analyse sera, en fait, que le traitement discursif de l'intertextualité est un indicatif du degré de complexité des textes.

Certains résultats n'étonneront personne: les classes sociales des personnages, par exemple, correspondent grosso modo au public lecteur visé: milieu universitaire dans Copies conformes; avocats, médecins et gens aisés dans Le poison dans l'eau; prostituées, sœur défroquée nymphomane, ex-danseuse nue et détective privé corrompu dans Douze suspects... pour un suicide. Dans ce dernier polar, il y a une exception: un avocat, mais celui-ci s'explique par la culpabilité de ce protagoniste. Les interactions entre les personnages varient grandement d'un texte à l'autre. Ainsi, elles sont simples et bien régulées dans Le roman d'Agatha: détective et assistant, détective et cliente, maîtresse et valet, bons et méchants; elles sont stéréotypées dans Douze suspects... pour un suicide: les femmes sont avant tout des objets sexuels, il n'y en pas une dans le roman qui ne tente de séduire un homme et ces derniers ne pensent qu'à ça, bien entendu. Dans Meurtres à blanc, il y a somme toute très peu d'interactions, les personnages sont d'abord seuls et cherchent à communiquer sans jamais y parvenir: il y a Marie qui n'arrête pas d'attendre ses messages secrets et qui sombre dans la folie; et il y a Caroline qui recherche Mustapha et Abdul dans son Maroc de pacotille. Les interactions dominent, par contre, dans les deux autres romans. Dans Le poison dans l'eau, l'intrigue se développe sur un fond d'inceste, de séparation, de haine longtemps refoulée et de désir. Il y a le père et la fille, le frère et la sœur, la belle-sœur et le mari, le père et la mère, la femme et le voisin, la détective et l'ami - chacune de ces relations constitue un noud dans le fil du récit. Quant à Copies conformes, aux relations déjà fort complexes qui lient les personnages du roman se superpose la trame encore plus trouble de l'intrigue du Maltese Falcon, de Dashiell Hammett. 
Les niveaux et les jeux de langage varient, de la même façon, avec les textes. Le jeu le plus coloré se retrouve dans Meurtre à blanc, où le registre des couleurs est développé à fond. Jusqu'à 170 mentions de couleurs sont présentes dans le texte, celui-ci virant du bleu au rouge, en passant par le noir, le vert, le bronze et le mauve. Le jeu atteint son paroxysme au deuxième chapitre où brillent 73 mentions de couleur, dont 30 "rouge" .

Quant aux niveaux de langage, le contraste peut-être le plus important oppose Douze suspects... pour un suicide, destiné à un public... adulte, et Le roman d'Agatha, destiné aux 10-12 ans. Le premier est écrit dans un français plus qu'approximatif. Quand, par exemple, une des suspectes offre un cognac au Manchot, elle sert l'alcool non pas dans des ballons mais dans "deux verres énormes, fabriqués spécialement pour y mettre cette eau de vie" (p. 60); plus loin, le Manchot va se servir de son "télé-chasseur (bell-boy) pour communiquer avec ses adjoints" (p. 117). Cela contraste avec Le roman d'Agatha, qui, lui, regorge de "gris anthracite", "ton goguenard ", "caban vermoulu", "linteau de cheminée " et autres "candélabres" de même lustre. En fait, de tous les romans, c'est dans celui-ci que le vocabulaire est le plus recherché. Les jeux de mots y sont d'ailleurs légion, dans les titres de chapitres, entre autres, où l'on retrouve des perles telles que: "Quel crime trame-t-on?", "Dites-le avec une brique", "Par l'auteur alléché", "Un œil-de-bøuf vachement juste" et le très bilingue "Faux rêveur pour toujours". Nous sommes loin des titres fonctionnels et plats de Saurel et de son Manchot: * Ia main du mort", "Des femmes suspectes", "Une nuit mouvementée" et "Le manchot triomphe. D'ailleurs, ce roman de Saurel brille non seulement par ses approximations et banalités lexicales, mais par une incohérence fondamentale: les personnages sont inconséquents, le jeu sur le suicide du détective, qui donne son titre au roman, est malhabile, les contradictions abondent. Le roman se lit, mais il se "consume" subrepticement au fur et à mesure qu'on y progresse, de sorte qu'il se défait totalement lors d'un retour en arrière ou d'une lecture seconde. En fait, sa cohérence est à ce point faible que la seule qu'on puisse lui attribuer n'est qu'un effet de lecture première, cette impression qui provient du présupposé de cohérence que l'on applique machinalement à tout roman conventionnel, populaire ou non.

Les différences entre les livres du corpus sont multiples, je ne les passerai pas toutes en revue. Pour en illustrer le caractère 
100

cependant, je vais m'attarder aux jeux intertextuels qu'ils contiennent. Les cinq romans ont tous en effet un intertexte policier et sa nature et fonction correspondent tout à fait au degré de complexité du texte et à son statut institutionnel. Il va de la simple mention, qui permet un véritable raccourci de la mise en intrigue, à la symbiose, qui participe donc activement à la structuration de l'intrigue. Je vais montrer brièvement comment ces textes gèrent leur intertexte. On verra que plus les textes s'éloignent du genre et plus cet intertexte devient important.

L'intertexte policier le plus simple se retrouve dans Le poison dans l'eau de Chrystine Brouillet. L'intrigue de ce roman est d'ailleurs la plus conforme au genre. On y trouve une seule référence explicite au polar, un clin d'œil qui ne porte pas à conséquence. Maud Graham, l'inspectrice, se présente chez Emma, qui est amoureuse de son frère et qui a confessé dans une lettre le meurtre de sa belle-sœur, au moment même où celle-ci s'apprête à partir.

Emma fait entrer l'inspectrice en la félicitant de sa chance: si elle avait appelé deux heures plus tard, elle aurait trouvé le nid vide. C'est ainsi qu'on dit dans les romans policiers quand les criminels se sont échappés? Graham sourit: "Je ne sais pas; je n'ai jamais remarqué.. (p. 143)

À qui s'adresse ce sourire de Graham? À qui s'adressent ses propos? À Emma ou au lecteur? La référence au discours du roman policier a une portée limitée. C'est un jeu anodin, isolé, sans conséquence. Il ne s'agit pas d'un procédé métatextuel, qui favoriserait le passage d'une attention de lecture à une autre, de l'univers narratif au texte qui l'exprime par exemple; mais au contraire, un simple bon mot suivi d'une répartie intelligente. Le texte de Brouillet est un roman conventionnel - ce n'est pas une métafiction postmoderne -, et cette référence au polar s'avère un dû, un clin d'œil obligé au genre.

Dans Douze suspects... pour un suicide, l'intertexte policier joue le rôle d'une prothèse narrative. Comme le signale le titre, un des traits de l'intrigue est l'accumulation de suspects pour un crime contre la personne qui n'engage habituellement que la victime elle-même. Or, pour atteindre ce chiffre, le texte va devoir tricher un peu. Les premiers suspects s'imposent facilement. Un détective privé véreux, Hector Riendeau, est retrouvé mort dans son bureau; c'est avec son arme qu'on a tiré, elle porte ses empreintes digitales. Est-ce un suicide déguisé en meurtre ou 
un meurtre déguisé en suicide? Le cadavre a été découvert par un avocat et son client que le détective faisait chanter. En voilà donc deux. Le mort venait, de plus, de prendre une importante assurance sur la vie. Sa femme, Yvonne Riendeau, attend le chèque. C'est la troisième. Son père, Onil Breton, est venu la retrouver; il est le quatrième. Et puis, il y a tous ceux qui avaient une dent contre lui, qui avaient loué ses services uniquement pour le voir se retourner contre eux et les faire chanter. Une bonne demi-douzaine. Pourtant, dans toute cette masse de suspects, il y en a peu de crédibles, sauf le client de l'avocat, déjà surveillé de près. Comment faire pour déplacer l'attention du lecteur loin du premier suspect, pour en isoler un autre? C'est facile, en le désignant explicitement. Le père de la veuve est un charmant ivrogne? Excellent, c'est le suspect idéal... Cette désignation pas trop rigide va se faire en deux temps trois mouvements. Le Manchot va expliquer: "Onil Breton est très intelligent. Il vient à peine de se réconcilier avec sa fille. [...] le bonhomme n'a pas d'alibi. Il est allé au cinéma, donc, impossible à vérifier. " (p. 97) À cela, un des assistants du Manchot répond, et c'est là que l'on retrouve la référence au polar: «Dans tous les romans policiers que j'ai lus, c'est le personnage le plus sympathique qui est coupable. " (p. 97) C'est l'argument d'autorité. Plus le père sera sympathique, et plus il sera suspect. Le tour est joué. Plutôt que de montrer le père en coupable, ce qui serait long, le texte prend donc un raccourci et le désigne via les lois du genre. Voilà un intertexte des plus fonctionnels.

Le roman d'Agatha d'Yves Arnau, malgré la jeunesse de son public et la simplicité de son intrigue, est à ce titre beaucoup plus inventif. Les références au roman policier y sont nombreuses et elles participent pleinement au jeu du texte. Le roman reprend sur le mode parodique les grands traits du roman policier: la relation détective/assistant, l'incarnation du méchant en une "organisation ", avec un mystérieux numéro Un, l'enquête avec des raisonnements à faire frémir Harry Dickson, un valet qui est coupable, une vieille dame aux abois, et ainsi de suite. Cette intertextualité passe aussi par l'onomastique, à commencer par le nom propre du détective privé, Edgar Allan, qui renvoie à Poe. Il y a aussi Agatha Grisly, la romancière, dont les polars ont été traduits dans plus de *dix-huit langues et dialectes . (p. 39) et qui en est à son quatre-vingt-sixième roman! Bien sûr, en lisant ce nom, on pense aussitôt à Christie, mais, nous dit le texte, cela n'a rien à voir: 
Notre Agatha a nous est en fait une petite Bellehumeur, des environs de Trois-Rivières, Grisly est un nom de plume qu'elle a choisi en hommage à son grand-oncle Max, trappeur et chasseur d'ours dans les Rocheuses de 1822 à 1839. C'est dire à quel point la grande Agatha Grisly est une petite fille bien de chez nous! Trifluvienne pure laine! (p. 40)

Le valet de la dame s'appelle Alfred Thicock et le détectivepersonnage de ses romans, rien de moins qu'Ulysse Rhubarbe, un nom à la hauteur d'Hercule Poirot. Les références ne s'arrêtent pas au polar: La Fontaine, Alexandre Dumas, Rambo, Frankenstein sont aussi interpellés. Ce roman est même, comme tout bon roman postmoderne, le lieu de quelques mises en abyme, d'une prolifération de romans policiers. Le premier est celui que le jeune lecteur lit, dont le titre est Le roman d'Agatha. Le second est celui qu'écrit justement la romancière Agatha Grisly, intitulé Cherche pas à savoir, mon coco! C'est ce roman que l'organisation malfaisante veut voler. Comme les malfaiteurs savent que la romancière ne relit jamais ce qu'elle écrit, ils lui volent, la nuit, les chapitres terminés qu'ils remplacent par des feuilles qui ne veulent rien dire. C'est le troisième texte, un faux roman policier. Le quatrième et dernier est l'œuvre du valet, Alfred Thicock, qui veut égaler sa maitresse et écrire à son tour un polar. Il croit ainsi décrocher le prix Nobel de littérature. Ce roman n'a pas de nom, mais il a pour héros Edgar Allan et son assistant, que le valet suit à la trace depuis leur arrivée et dont il s'inspire. Tous ces romans s'emboîtent les uns dans les autres, dans une intrigue pourtant adaptée aux 10 à 12 ans.

Meurtres à blanc, de Yolande Villemaire, ne fait pas dans le tape-à-l'œil avec la même verve, quoique dans ce roman aussi les références soient nombreuses. La première apparaît dès le premier paragraphe et elle a une valeur programmatique. Cette référence survient à point, elle vient renforcer le caractère paradoxal de l'entrée en matière. Voici l'incipit:

J'écris. Point. Une vague Caroline (oh! le joli nom!) marchande un collier flou (supposément en bronze) dans un souk de Tétouan. À l'heure de la sieste. J'écris et je fume des Export A régulier et la maudite clochette de fin de ligne me fait sursauter à tout coup. J'ai essayé de passer le temps en relisant l'Agatha Christie que j'ai lu dans le train mais, rien à faire; celui-là, comme tous les romans policiers, n'a pu résister à une seconde lecture. (p. 10)

C'est un début tout en rupture. La première s'inscrit même dès les deux premières phrases du texte. C'est le "J'écris. Point." 
Il y a là un message paradoxal du même type que "Soyez spontanés " ou encore l'affiche qui dicte de ne pas afficher. "J'écris. Point. ": ce qui se présente comme une écriture porte au contraire des traces d'oralité. La seule situation où l'on utilise le terme "point" survient quand, en parlant ou en dictant, on veut signaler la fin d'une phrase, fin qui n'est pas évidente. Pour s'en convaincre, il suffit de dicter cet incipit, ce qui donne, dans une accumulation intempestive de points: “j'écris (point) point (point). C'est un texte écrit mais oral. Ce qui semble être confirmé par les parenthèses et les exclamations *(oh! le joli nom!)", les hésitations ou improvisations de la narratrice ("une vague Caroline", supposément), l'absence de cohésion des phrases (on passe de la machine à écrire au Maroc, de l'écriture à la lecture), et même le nom de la ville marocaine, Tétouan, très près d'un impératif "tais-toi *.

C'est donc dans un tel contexte qu'apparaît la référence à Agatha Christie et à ses romans. Le jugement de la narratrice est clair: les romans policiers ne résistent pas à une seconde lecture. Si cette affirmation vaut la peine d'être répétée, c'est qu'elle s'applique avant tout à Meurtres à blanc, d'où sa nature programmatique. Mais, à l'encontre des romans d'Agatha, celui de Villemaire ne résiste pas, lui, à une première lecture: il se met à résister à partir de la seconde. Et cette résistance fait partie de sa mise en intrigue. On relit difficilement un roman policier conventionnel, simplement parce qu'on en connaît la fin et le coupable, et que les ficelles d'abord invisibles sont devenues beaucoup trop grosses. On ne peut pas faire semblant de ne pas savoir, ce qui ôte tout le plaisir. Mais la situation avec Meurtres à blanc est inversée. Connaître la fin du texte incite le lecteur non pas à fermer le livre pour en choisir un autre, mais à le relire depuis le début, à passer d'une lecture avant tout axée sur la progression à travers le texte à une lecture axée sur sa compréhension ${ }^{9}$. Et c'est dans ce mouvement de retour que le lecteur redécouvre l'affirmation sur les polars qui, plus qu'une boutade, apparaît dès lors comme un véritable avertissement: * il ne sert à rien de repasser à travers le texte, on ne trouvera pas ce qu'on y cherche."

Le roman appelle une seconde lecture, vouée malheureusement à l'échec. Une lecture avant tout policière, une recherche

Bertrand Gervais, «ecture: tensions et régies*, Poétique, Paris, Seuil, $n^{\circ} 89,1992$, p. 105-125. 
d'indices, de traces, de symptômes: ceux de la décomposition de la narratrice, de la progression de sa folie, de son dédoublement, de sa perte d'identité. Marie, la narratrice-espionne, invente pour passer le temps entre deux messages secrets le récit d'une Caroline, au Maroc, impliquée dans des histoires un peu louches. Le texte oscille entre ces deux situations narratives, qui se répondent l'une l'autre. Caroline est le personnage de Marie, son récit est sa fiction. Mais à la toute fin, dans un retournement, on apprend, par Caroline, la mort de Marie. Caroline ne serait pas un personnage mais bel et bien la soeur de Marie et son retour du Maroc a précipité certains événements. Mais cet aveu de Caroline n'est pas aussitôt achevé que Marie, ressuscitée d'entre les mortes, reprend la parole pour nier l'existence de cette Caroline qui n'a jamais été plus que l'héroïne d'un roman inachevé. Le roman ne se termine donc pas sur une explication qui viendrait boucler l'intrigue, dans la grande tradition des polars, mais sur une série de ruptures, qui rendent le mystère encore plus dense ${ }^{10}$. Le lecteur qui veut comprendre plus doit rebrousser chemin et se mettre à la recherche des traces annonciatrices de cette déconstruction. D'où la pertinence de l'avertissement.

Le plat de résistance intertextuel est Copies conformes de Monique LaRue. Il ne s'agit pas en soi d'un roman policier - il n'y a pas à proprement parler de détective ni même d'enquête mais d'un roman dont la structure, l'intrigue, le cadre, les personnages et les thèmes sont inspirés du roman noir américain. Pas de n'importe quel roman, mais du plus célèbre de Dashiell Hammett, The Maltese Falcon.

Le roman de LaRue n'est pas une copie conforme du roman de Hammett; il est plutôt, et de façon plus complexe, en symbiose avec lui. L'intrigue ne tourne pas autour d'une petite statuette que tous cherchent à récupérer, mais d'une disquette, d'une plaquette de Bernouilli qui a été subtilisée. La plaquette appartenait au mari de la narratrice, Claire Dubé, qui a dû repartir en catastrophe pour Montréal, et celle-ci doit la récupérer avant son propre retour, dans six jours à peine. Le cadre du roman est Berkeley et San Francisco, là où justement se déroulait l'intrigue du Maltese Falcon, mais la distribution n'est pas tout à fait la

10 Une telle fin semble inspirée de L'antiphonaire d'Hubert Aquin (Montréal, Cercle du livre de France, 1969), qui connaît une fin tout aussi problématique. 
même. Disparus les Sam Spade, Effie Perine et Miles Archer, les Joel Cairo, Casper Gutman et le petit Wilmer Cook. Ils ont été remplacés par des Claire Dubé, Alain Vasseur, Ron O'Doorsey, Diran Zarian et Bob Mason, un peu plus modernes. Une chose cependant semble ne pas avoir changé, c'est le personnage de Brigid, qui se retrouve dans les deux univers. À la Brigid O'Shaughnessy du Maltese Falcon, correspond dans Copies conformes une Brigid O'Doorsey. Une Brigid encore une fois au centre de tout, qui se défile comme dans ses beaux jours, qui ment, ruse, et se trouve mêlée à toutes sortes d'histoires. Comme la première, elle s'enfuit avec un bien précieux, cette fois le fils de son mari, et elle incarne la figure de la traîtresse. Comme la première, elle s'habille surtout en bleu, elle est très belle et porte ses cheveux «fauves" et «bouclés" (p. 102). Pourtant, ce n'est pas une copie conforme; car Brigid a beaucoup vieilli, elle s'est émaciée, a séché sur place comme une momie, à coup de chirurgie plastique et de diètes sévères: "Une droguée du scalpel." (p. 74) Elle ne fait plus dans le recel de statuettes, mais dans le rapt de plaquettes.

C'est donc par elle que se nouent les liens entre les deux univers. Or, ces liens, elle ne les subit pas mais, au contraire, les recherche activement. Brigid, tout comme son frère Ron O'Doorsey, voue au Maltese Falcon un véritable culte. Il est question pour la première fois de ce roman à la page 20. C'est Ron O'Doorsey qui explique à Claire Dubé ce que vend leur compagnie (ce n'est pas tout à fait le premier intertexte policier du roman; un peu plus tôt, il avait été question d'une voisine du nom de Miss Marple; p. 17):

Brigid et moi avons fondé une compagnie: The Maltese Falcon Inc. Nous vendons des jeux électroniques par correspondance. Notre plus grand succès est inspiré du roman de Dashiell Hammett. Il s'agit de trouver un faucon. On décide au hasard qui sera Brigid O'Shaughnessy, Sam Spade. C'est un jeu de rôle. Le gagnant est celui qui trouve le vrai faucon... (p. 20).

En fait, plus qu'un simple jeu de rôle, le roman de Hammett agit dans ce texte comme un véritable virus informatique. La comparaison est appropriée parce qu'elle reprend un des thèmes centraux du roman: l'informatique et les logiciels qui servent à copier, à traduire, à jouer. À partir de cette première mention, le roman de Hammett va occuper un rôle de plus en plus important dans le récit, il va réussir à s'infiltrer dans toutes les composantes 
de l'intrigue et à prendre toute la place. Il va servir à la fois de source thématique (sur l'amour, le désir), de structure narrative (la recherche d'un objet volé), de modèle de comportement (pour Brigid, mais aussi pour Claire Dubé, dans un savant jeu de miroir), de point de repère et de guide touristique pour San Francisco, de sujet de conversation, et même d'algorithme de résolution. C'est grâce à ce roman que Claire Dubé réussit à retrouver la trace des O'Doorsey. Elle s'en sert comme d'une carte dans un signe de piste, accumulant les associations, les recoupements, les interprétations. À son contact, elle se transforme en détective, menant l'enquête, interprétant les événements à la lumière des péripéties de Sam Spade et de Joel Cairo. À sa lecture, elle rejoint enfin les O'Doorsey, retrouve la plaquette de Bernouilli de son mari, de même que l'argent qu'on lui devait. The Maltese Falcon s'est répandu partout, comme un virus. Soixante ans plus tard, les faucons sont peut-être disparus, remplacés par des puces, mais les mêmes soifs persistent, les mêmes désirs.

Après ce bref survol, on comprend que l'intertexte policier est géré de façon bien différente d'un roman à l'autre: du virus à la simple remarque, de la béquille à l'avertissement. Les rôles de cet intertexte sont variés, ils semblent pourtant remplir la même fonction, celle d'assurer à ces textes des liens avec un genre dont ils sont à peu près tous à la périphérie. L'éloignement rend nécessaire la mention. Quant au scalaire, il ne s'agit là que d'une seule de ses manifestations. Elle montre bien pourtant quelles peuvent être les différences de complexité des textes et comment celles-ci impliquent des compétences et des attentions de lecture à leur tour différentes. Mais cela ne doit pas surprendre outre mesure. Aucun roman n'appelle la même attention de lecture (il suffit, pour s'en convaincre, de comparer le polar de Saurel, qui requiert presque une inattention au texte, qui seule permet de ne pas être trop dérangé par ses incohérences, au roman de LaRue, qui demande au contraire d'être très attentif aux jeux entre les deux intrigues croisées et aux transformations opérées, ou encore au roman d'Arnau, dont l'accumulation des procédés discursifs met à rude épreuve l'attention de son jeune lecteur); aucun, quel que soit son degré de complexité, ne se lit de la même façon. 